\title{
THE EFFECTIVENESS OF EXTRACORPOREAL SHOCK WAVE THERAPY ON BREAST CANCER-RELATED LYMPHEDEMA: A LITERATURE REVIEW
}

\author{
S. Miccinilli, M. Bravi, M. Maselli, F. Santacaterina,
} M. Morrone, D. Manco, R. Toglia, S. Sterzi, F. Bressi

Physical and Rehabilitative Medicine, Università Campus Bio-Medico di Roma, Italy

\section{ABSTRACT}

Lymphedema is one of the most dreaded complications related to breast cancer surgery, commonly resulting in upper limb functional, esthetic, and psychological impairment. The necessity to improve the efficacy of conventional treatments and the promising effect of extracorporeal shock wave therapy (ESWT) on lymphangiogenesis in vitro and animal models, has prompted studies involving women affected by breast cancer-related lymphedema. Since intervention modalities and treatment protocols used are different, a review is necessary to verify the effectiveness of ESWT, evaluating the quality of existing studies and the eventual need for further research. Data were obtained from PubMed, Scopus, Google Scholar, Cochrane Library and PEDro, including articles published until January 2019. Five studies met the inclusion criteria. Evident heterogeneity emerged among selected studies permitting only a purely descriptive analysis of their data and strongly limiting their comparison. When compared to other treatment modalities, ESWT showed a significant effect on measured outcomes. It is clear that further high quality research is necessary to assert with confidence the effects and possible superiority of ESWT over other conservative therapies in the management of breast cancer-related lymphedema.

Keywords: extracorporeal shock wave therapy, lymphedema, physical medicine and rehabilitation, breast cancer

Lymphedema is one of the most dreaded complications related to breast cancer surgery. It commonly results in upper limb functional, esthetic, and psychological impairment, thereby affecting the quality-of-life of women who develop breast cancer-related lymphedema. This pathological condition is characterized by an abnormal collection of high-molecular-weight protein fluid in the interstitium, as a result of functional overload of the lymphatic system. Lymphatic system overload is due to a greater volume of lymph than the lymphatic transport capability, which is impaired following axillary dissection, often enhanced by coexisting factors. These coexisting factors may include the extent of surgery, the patient's age, history of obesity, infection, and receipt of adjuvant treatments. The incidence of lymphedema in breast cancer patients has been reported to be between $0 \%$ and $77 \%$ (1). Moreover, the risk of lymphedema with axillary lymph node dissection (ALND) averages $28 \%$, varying over time and depending on type of the clinical measurements and of the surgery. It mostly presents by three years but this may continue over a lifetime.

\section{Description of the Condition}

Lymphedema can be classified as a 
primary or a secondary disease. In breast cancer patients, it occurs as a secondary pathological condition as a consequence of axillary surgery and/or radiation therapy. The pressure in the initial lymphatic vessels is usually negative and after ALND, intralymphatic pressure is affected and lymphatic flow becomes slower than normal. Therefore, the normal function in returning proteins, lipids, and water from the interstitial to the intravascular space is reduced, leading to increased accumulation of the interstitial fluid and the stagnation of high-molecular-weight proteins. This accumulation of proteins and fluids is first a transitory phase, lasting one to three weeks, then leading to a latent phase which can last from four months to years and may not coincide with clinical signs and symptoms. The accumulation of interstitial fluid progressively leads to dilatation of the remaining outflow tracts that causes reversal of flow from subcutaneous tissues into the dermal plexus. This latter event leads to fibrosis and fat accumulation and provides an excellent culture medium for bacteria to grow, also promoting a marked inflammatory reaction that causes a change from the initial pitting edema to the brawny non-pitting edema characteristic of lymphedema.

Based on its timing, lymphedema can also be classified as acute or chronic. Acute breast cancer lymphedema generally occurs within six months after mastectomy and lasts three to six months, usually as a pitting, transient, and self-limited upper limb edema. Chronic lymphedema is instead present for at least three months, described as a non-pitting upper limb edema associated with skin changes and high risk of developing ulcers and infections. All these observations lead to the evidence that lymphedema is a progressive condition, as described by The International Society of Lymphology Staging System (2):

Stage 0 - latent or subclinical (despite the impaired lymph transport, swelling is not yet clinically evident);

Stage 1 - early accumulation of fluid (the tissue swelling is clinically evident, it subsides with limb elevation, pitting may occur);

Stage 2 - advanced accumulation of fluid (limb elevation alone rarely reduces swelling, pitting is manifest, until lately tissue fibrosis supervenes);

Stage 3 - lymphostatic elephantiasis (pitting is absent and trophic skin changes such as acanthosis and adipose hypertrophy develop).

Within each stage, the severity of lymphedema can be classified based on limb volume differences: minimal lymphedema ( $<20 \%$ increase), moderate lymphedema (20\%-40\% increase), or severe lymphedema (>40\%) (1). An accurate history and a thorough physical examination are necessary to establish the diagnosis of lymphedema, and several approaches have been attempted to detect and follow patients' progression and/or response to therapy. These objective tech-niques include measurements of limb circum-ferences, water displacement, ultrasound, tonometry, optoelectronic perometry, and bioimpedance. Lymphoscintigraphy, magnetic resonance imaging (MRI), and computed tomography (CT) may be necessary particu-larly in difficult cases. Concerning the management of breast cancer-related lymph-edema, it should first include adequate patient education with respect to activity levels and infection prophylaxis. Complete or Complex Decongestive Physiotherapy/Therapy (CDP/T) is considered the standard of care $(1,2,4,5)$. It comprises two phases of treatment: the first phase lasts four weeks, consisting of four different steps (skin care, manual lymphatic drainage, compression bandaging and physical therapy exercises); the second phase consists instead in applying low resistance short-stretch compressive bandages, to enhance and maintain lymphatic drainage. Finally, surgical treatment should be reserved for patients with long-term complications or previous medical treatment failure.

\section{Description of the Intervention}

A statement on what ESWT is and why it is relevant in LE is needed (for example, how could ESWT promote lymphangiogenesis). Further, several in vitro and/or animal model studies have provided preliminary evidence 
that support that ESWT may benefit lymphedema. Specifically, Kim et al (6) evaluated the therapeutic effects of ESWT applied in post-surgical lymphedema in a mouse model, when compared and then combined with administration of VEGF-C hydrogel. The authors observed clinical improvement of lymphedema in each experimental group, however with a greater decrease of edema in the association therapy group. Investigating the effects of low-energy ESWT applied in secondary lymphedema in a rabbit ear model, Kubo et al (7) observed attenuation of the lymphedema, together with a heightened expression of VEGF-C, VEGFR3, and lymphangiogenesis. Finally, Serizawa et al (8) examined whether lowenergy ESWT improved lymphedema in a rat model and if VEGF and bFGF were involved in the process. The study showed not only a statistically significant improvement in the considered outcome due to the application of ESWT but also a connection of this result to increased expression of the aforementioned factors. Moreover, from the 1990's on, ESWT has been successfully used to treat musculoskeletal disorders with evidence of stimulating several important healing processes, including local revascularization and inflammatory-mediated response (9). These positive, albeit preliminary results have paved the way for evaluating ESWT in human studies. Thus, a review of the literature is now necessary to summarize what has been evaluated to date, the quality of these studies and the eventual need for further research, giving clearer answers about efficacy of ESWT in the treatment of breast cancer-related lymphedema.

\section{Objectives}

The main purpose of this review is therefore to explore the effectiveness of ESWT in improving clinical and functional outcomes in subjects affected by breast cancer-related lymphedema. The secondary objective is to identify any biases in the scientific literature and give direction to further research on ESWT's application in the treatment of this disorder.

\section{MATERIALS AND METHODS}

Criteria for considering studies for this review:

Types of Studies

Randomized controlled trials (RCTs) and prospective study designs in any language were included in this review. All the included studies investigated the effects of shockwave therapy in the treatment of breast cancer- or not related lymphedema.

\section{Types of Participants}

Studies on adult subjects ( $>18$ years) affected by breast cancer-related lymphedema were included. Breast cancer-related lymphedema was defined as a clinical syndrome characterized by increase in volume, circumferences, or skin thickness on the upper limb of the affected side, often associated with shoulder range of motion (ROM) and functioning reduction (1).

\section{Types of Interventions}

All studies comparing ESWT with placebo or other treatment modalities with different types and dosages of shock wave therapy were included.

\section{Types of Outcome Measures}

Upper limb volume and circumferences, shoulder ROM and function, skin characteristics (e.g., thickness, hardness), sensory impairment, handgrip strength, and quality of life. Since there is no standard clinical outcome protocol for upper limb lymphedema, all the outcome measures reported in the selected trials have been taken into consideration in our review.

\section{Search Strategy}

Our research question was formulated using PICO (Population, Intervention/ 
TABLE 1

The PICO Formatted Question for Analysis

\begin{tabular}{|l|l|}
\hline Population & $\begin{array}{l}\text { Adults aged 18 years or older, affected by Breast Cancer-related } \\
\text { Lymphedema }\end{array}$ \\
\hline Intervention/Exposure & Extracorporeal Shock Wave Therapy \\
\hline Comparison & Any Conservative treatment or sham \\
\hline Outcome & $\begin{array}{l}\text { Upper limb volume, circumferences, skin thickness, functioning and } \\
\text { shoulder ROM }\end{array}$ \\
\hline
\end{tabular}

Exposure, Comparison, and Outcome) framework (Table 1). A search of scientific studies concerning the use of shockwave therapy in the treatment of breast cancerrelated lymphedema was performed in PubMed/Medline and Google Scholar electronic databases using the following keywords "Lymphedema and shock wave therapy." The same search was conducted in Cochrane Library using the keywords "Lymphedema and shock wave." Search in PEDro database was also performed using the keywords: "Lymphedema and extracorporeal shock wave therapy." We chose these keywords to include in our research both pathology and treatment.

\section{Data Collection and Analysis}

\section{Eligibility criteria}

All the included studies investigate the effects of shockwave therapy on breast cancerrelated lymphedema. In particular, they fulfill the following criteria: a) patients affected by breast cancer-related lymphedema were included, b) shockwave therapy was evaluated as a choice treatment of breast cancer-related lymphedema, c) results for upper limb volume/ circumferences and/or ROM and/or functional outcomes were reported. Articles not written in English were not examined due to linguistic barriers. No limits either for time, status of publication, or for length of follow up were applied to our search.

\section{Information sources}

The last search was conducted on the 7 th of January 2019.

\section{Study selection}

To determine eligibility, each single article emerged from the literature search had been analyzed by its title, abstract and full-text with two reviewers independently applying the inclusion criteria to select relevant studies. Discussion was the consensus method used to solve differences in opinions concerning inclusion of studies.

\section{Data extraction and synthesis}

Data collection was organized in several steps. The first step was to extract from each study the information about: population studied (age, duration of symptoms), shockwave treatment (modality features and parameters), control group (if present) treatment, other interventions, outcome measures, and results. The extracted data were arranged in a tabular form to facilitate the double-check by the second author. Authors discussed together the relevance of findings and results.

\section{Methodological quality assessment}

The methodological quality assessment of the included studies was performed by two 


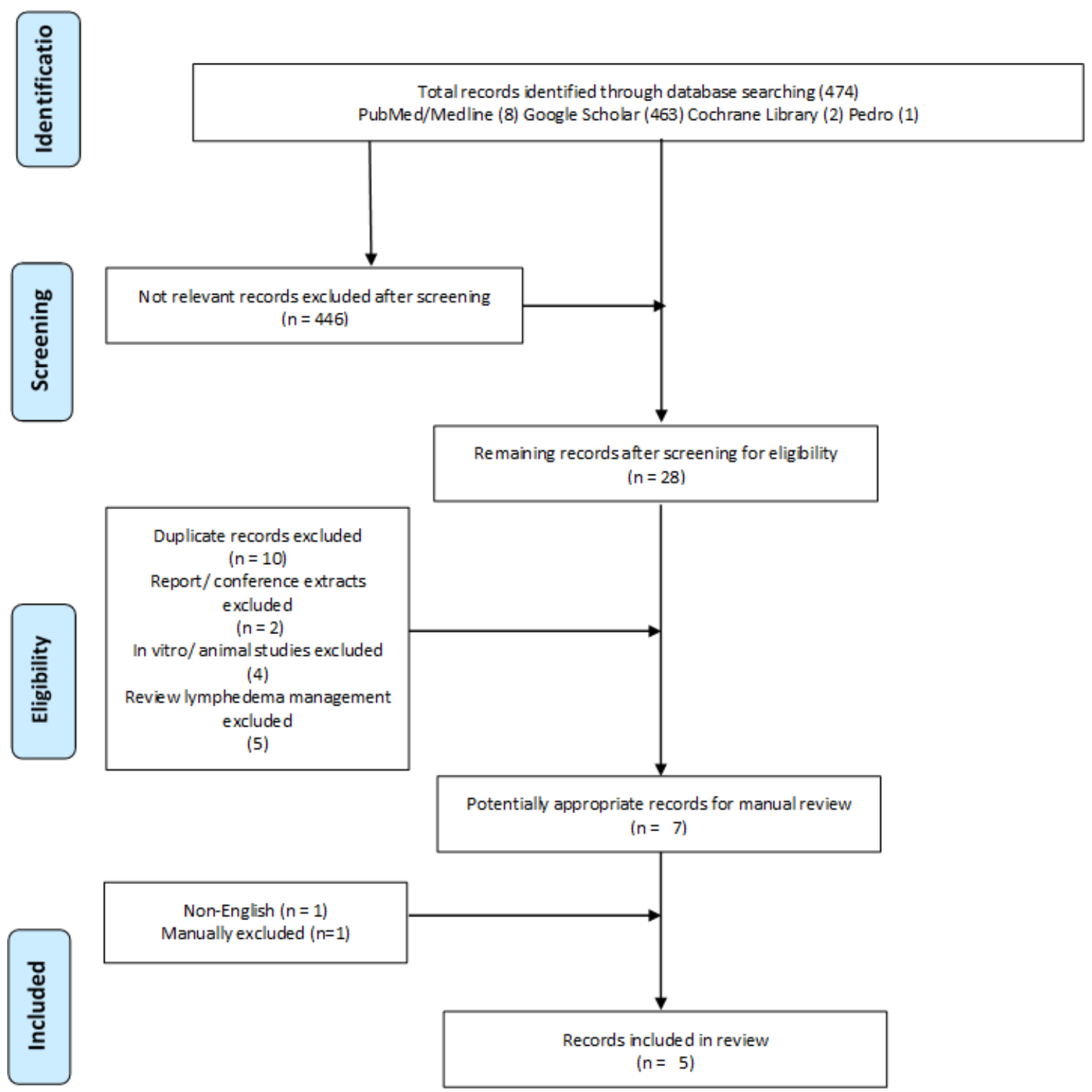

Fig. 1. Prisma Flow Chart for selecting studies for analysis.

different reviewers. The PEDro Scale and the Modified McMaster Quantitative Critical Appraisal Tool were used to examine quality, respectively, of randomized controlled trials (RCTs) and of non-RCTs. Discussion was the consensus method used to overcome different judgments.
Assessment of risk of bias in included studies

In order to appraise the risk of bias of each selected study, the Risk of bias Assessment Tool of the Review Manager - RevMan (version 5.3. Copenhagen: The Nordic Cochrane Centre, The Cochrane Collabora- 
TABLE 2

PEDro Scale Results for 3 of the Selected Studies

\begin{tabular}{|l|c|c|c|}
\hline PEDro Criterion & $\begin{array}{c}\text { Abdelhaim et al. } \\
2018(11)\end{array}$ & $\begin{array}{c}\text { El-Shazly et al. } \\
2016(12)\end{array}$ & $\begin{array}{c}\text { Mahran et al. } \\
\text { 2015 (13) }\end{array}$ \\
\hline Eligibility criteria & 1 & 1 & 1 \\
\hline Random allocation & 1 & 1 & 1 \\
\hline Concealed allocation & 1 & 1 & 1 \\
\hline Baseline comparability & 1 & 0 & 0 \\
\hline Blind subjects & 0 & 0 & 0 \\
\hline Blind Therapists & 0 & 0 & 0 \\
\hline Blind assessors & 1 & 0 & 0 \\
\hline Adequate Follow UP & 1 & 0 & 1 \\
\hline Intention-to-treat analysis & 0 & 1 & 1 \\
\hline Between-group comparisons & 1 & 1 & $4 / 10$ \\
\hline Point estimates and variability & 1 & $4 / 10$ & 40 \\
\hline Total & $7 / 10$ & 40 & 0 \\
\hline Percentage score & 70 & 0 & 0 \\
\hline
\end{tabular}

tion, 2014) was used.

\section{RESULTS}

Search Results / Descriptive Studies:

The PRISMA guidelines were used to select the studies. The screening and eligibility process is outlined in Fig. 1. A total number of 474 records was at first identified through databases searching. 28 titles of the initial selected records were included as they did satisfy the inclusion criteria. Upon first examination, 10 were duplicates, 2 were reports/conference extracts, 4 were in vitro studies, and 5 were reviews. Of the 7 potentially appropriate records, 1 was excluded because it was written in Russian and 1 was manually excluded because the purpose of the study was not appropriate for our PICO search strategy. Therefore, studies included in the review comprised 3 RCTs and 2 prospective pilot studies. As regards the studies quality, the PEDro scale was used to evaluate the included RCTs, and the obtained scores ranged between 40/100 and 70/100 (See Table 2 for details). The Modified McMaster Quantitative Critical Appraisal Tool, used to appraise quality of the two prospective pilot studies, instead showed respectively a score of 53/100 and 67/100 (See Table 3 for details).

\section{Interventions}

A variety of devices and different sets of 
TABLE 3

Modified MeMaster Quantitative Critical Appraisal Tool for 2 of the Selected Studies

\begin{tabular}{|c|c|c|}
\hline Criterion & $\begin{array}{c}\text { Cebicci et al. } \\
2016(10)\end{array}$ & $\begin{array}{c}\text { Bae et al. } 2013 \\
\text { (14) }\end{array}$ \\
\hline Study purpose stated clearly & 1 & 1 \\
\hline Relevant background literature reviewed & 1 & 1 \\
\hline Research design appropriate & 1 & 1 \\
\hline Sample described in detail & 1 & 1 \\
\hline Sample size justified & 0 & $\mathbf{0}$ \\
\hline Outcome measures reliable & 1 & 1 \\
\hline Outcome measures valid & 1 & 1 \\
\hline Intervention/exposure described in detail & 1 & $\mathbf{0}$ \\
\hline Contamination avoided & $\mathbf{0}$ & $\mathbf{0}$ \\
\hline Cointervention avoided & $\mathbf{0}$ & $\mathbf{0}$ \\
\hline $\begin{array}{l}\text { Results were reported in terms of statistical } \\
\text { significance }\end{array}$ & 1 & 1 \\
\hline Appropriate analysis methods & 1 & $\mathbf{0}$ \\
\hline Clinical importance reported & $\mathbf{0}$ & $\mathbf{0}$ \\
\hline Dropouts reported & $\mathbf{0}$ & $\mathbf{0}$ \\
\hline Appropriate conclusions & 1 & 1 \\
\hline Total & $10 / 15$ & $8 / 15$ \\
\hline Percentage score & 67 & 53 \\
\hline
\end{tabular}

shock wave parameters were used in the selected studies (See Table 4 for details). Only one of the selected trials specified the diameter of the used head and that each session was performed by the same physical therapist (10) while only two of the five trials specified that no local anesthetic was administered before treatment $(10,11)$. Three trials administered a total number of twelve treatments (10-12): two studies administered three treatments week for four weeks $(10,11)$; one study administered instead two treatments per week, over a period of six weeks (12). The other two studies both administered two treatments per week with one study administering a total number of sixteen treatments in 8 weeks (13) and the other administering instead only four treatments over a period of two weeks (14). 


\section{TABLE 4}

ESWT Treatment Parameters, Method of Application, and

\section{Other Interventions for Selected Studies}

\begin{tabular}{|c|c|c|c|c|}
\hline \multirow[b]{2}{*}{ Author } & \multicolumn{4}{|l|}{ Treatment } \\
\hline & $\begin{array}{l}\text { Shock wave } \\
\text { application }\end{array}$ & $\begin{array}{l}\text { Treatment } \\
\text { parameters }\end{array}$ & $\begin{array}{c}\text { No. of } \\
\text { sessions }\end{array}$ & $\begin{array}{l}\text { Interval } \\
\text { between } \\
\text { sessions }\end{array}$ \\
\hline $\begin{array}{l}\text { Abdelhalim et } \\
\text { al (2018) (11) }\end{array}$ & $\begin{array}{l}750 \text { for axillary lymph } \\
\text { nodes; } \\
250 \text { for cubital lymph nodes; } \\
1500 \text { for arm, forearm, hand }\end{array}$ & $\begin{array}{c}2500 \text { pulses; } \\
4 \mathrm{~Hz} ; \\
2 \text { bar; } \\
\text { No anesthesia }\end{array}$ & 12 & $\begin{array}{l}3 \text { times/week } \\
\text { for } 4 \text { weeks }\end{array}$ \\
\hline $\begin{array}{l}\text { El-Shazly et al } \\
(2016)(12)\end{array}$ & $\begin{array}{l}1000 \text { pulses for most fibrotic } \\
\text { areas } \\
1000 \text { pulses for less fibrotic } \\
\text { areas }\end{array}$ & $\begin{array}{c}2000 \text { pulse } \\
5 \mathrm{~Hz} \\
0.040-0.069 \\
\mathrm{~mJ} / \mathrm{mm}^{2} \\
10 \text { minutes }\end{array}$ & 12 & $\begin{array}{c}2 \text { times/week } \\
\text { for } 6 \text { weeks }\end{array}$ \\
\hline $\begin{array}{l}\text { Cebicci et al. } \\
(2016)(10)\end{array}$ & $\begin{array}{l}750 \text { for axillary lymph } \\
\text { nodes; } \\
250 \text { for cubital lymph nodes; } \\
1500 \text { for arm, forearm, hand }\end{array}$ & $\begin{array}{c}2500 \text { pulses; } \\
4 \mathrm{~Hz} \\
2 \mathrm{bar} \\
12 \mathrm{~mm} \text { head } \\
\text { No anesthesia }\end{array}$ & 12 & $\begin{array}{l}3 \text { times/week } \\
\text { for } 4 \text { weeks }\end{array}$ \\
\hline $\begin{array}{l}\text { Mahran et al. } \\
\text { (2015)(12) }\end{array}$ & Not specified & $\begin{array}{c}2500 \text { pulses; } \\
4 \mathrm{~Hz} \\
2 \mathrm{bar}\end{array}$ & 16 & $\begin{array}{c}2 \text { times/week } \\
\text { for } 8 \text { weeks }\end{array}$ \\
\hline $\begin{array}{l}\text { Bae et al (2013) } \\
\text { (14) }\end{array}$ & $\begin{array}{l}1000 \text { pulses for most fibrotic } \\
\text { areas } \\
1000 \text { pulses for less fibrotic } \\
\text { areas }\end{array}$ & $\begin{array}{l}2000 \text { pulses; } \\
0.056-0.068 \\
\mathrm{~mJ} / \mathrm{mm}^{2}\end{array}$ & 4 & $\begin{array}{c}2 \text { times/week } \\
\text { for } 2 \text { weeks }\end{array}$ \\
\hline
\end{tabular}

ESWT are applied to different areas of the affected upper limb. One of the selected trials (13) did not specify the site of ESWT application, two studies summarily reported that ESWT were applied respectively at the "most fibrotic" and "less fibrotic" point $(12,14)$, and two studies $(10,11)$ precisely described the site of application of the ESWT treatment (750 pulses at axillary lymph nodes, 250 pulses at cubital lymph nodes and 1500 pulses within arm, forearm, and hand regions).

Regarding the control group, a certain heterogeneity was observed in the proposed treatments. Three of the selected studies compared ESWT treatment with other conser- vative therapies (12-14). In particular, two studies compared the combination of ESWT treatment with traditional physical therapy to administering traditional physical therapy only $(12,13)$. In reference to the traditional therapy administered in the two aforementioned trials, El-Shazly et al (12) provided manual lymphatic drainage followed by circulatory exercises with elevation, shoulder ROM exercises, and pneumatic compression therapy (performed for $60^{\prime}, 3$ times a week), while Mahran et al (13) provided manual massage followed by circulatory exercises with elevation and compression bandaging (performed for 45 minutes, 3 times a week). Bae et al instead 
compared the effects derived from the use of ESWT treatment alone with those of the ESWT treatment in combination with lymphatic massage (14).

One study compared ESWT treatment with intermittent pneumatic compression therapy (comprehensive of a total of 20 sessions of 45 minutes with $60 \mathrm{mmHg}$ intermittent pneumatic compression applied to the affected arm, performed 5 times per week). Authors reported that both experimental and control groups observed also a daily home program exercises (including active ROM, pumping and elevation exercises for the affected upper extremity) and upper limb hygiene advice (e.g., avoiding sleeping on the affected side, preventing injury of the affected upper extremity) (11).

Finally, the prospective clinical pilot study performed by Cebicci et al reported effects derived from the use of ESWT treatment only but without a control group (10).

\section{Study participants}

All the selected trials recruited patients affected by breast cancer-related upper limb lymphedema. Only two of the selected trials outlined the stage of lymphedema in the recruited patients: El-Shazly et al recruited patients with lymphedema stage II and III (12); Bae et al. recruited patients with lymphedema stage III (14). Authors also specified certain cut-off limits for the inclusion of participants. Two studies required an affected side to non-affected side difference of 2 to 8 $\mathrm{cm}(11,13)$. Mahran et al also required a history of lymphedema of at least ten months elapsed since axilla node dissection (13). One study included patients with a volume difference greater than $200 \mathrm{ml}$ and a circumference difference $\geq 2 \mathrm{~cm}$ between affected and non-affected side (10). One study recruited patients with a limb-to-limb difference $\geq 2 \mathrm{~cm}$ at a single site measurement and lymphatic obstruction confirmed by lymphoscintigraphy (14). Two studies included patients who did not undergo any other lymphedema conservative treatment within a 6-months period $(10,11)$. These both required also that patients completed their chemo/radiotherapy treatment within no more than, respectively, 6 weeks (11) and 6 months (10). Mean age of study participants was similar in all selected trials (See Table 5 for details). Exclusion criteria were generally homogeneous: evidence of metastasis, infection, anomaly of vessels, hyperventilation, and polyneuropathy were the most used.

\section{Timing follow up}

Follow up assessment was performed at differing time points among the selected trials. (See Table 6 for details). Three studies performed follow up assessment only immediately after completion the treatment $(11,12,14)$. One study performed assessments immediately after completion the treatment and at one, three, and 6 months after treatment (10). One study finally performed assessment at 4 weeks after the first treatment (during treatment) and after completion of the whole treatment (13).

\section{Outcome assessment}

Only one study (11) specified that all assessments were conducted by the same evaluator, who was blinded to the intervention. The most used outcome measure was arm volume. Regarding arm volume, the aforementioned authors used water volume displacement as the measure of its evaluation, showing a certain variability in the method used. El-Shazly et al did not provide any specific information (12), Mahran et al measured the volume of water in millimeters displaced by the arm with lymphedema compared to the unaffected limb (13), Bae et al measured it using the derivation of Archimede's principle (14), and Cebicci et al applied the Tracey's volume category (10). Finally, only one of the selected studies did not evaluate this outcome (11).

The arm circumference measurement was the second most used outcome measure and evaluated in three of the selected studies 
TABLE 5

Characteristics of Participants in the Analyzed Studies

\begin{tabular}{|c|c|c|c|c|}
\hline Author & $\mathbf{N}$ & \multicolumn{2}{|c|}{ Age, mean \pm SD } & Duration of symptoms, months, \\
\hline $\begin{array}{l}\text { Abdelhalim et al } \\
\text { (2018)(11) }\end{array}$ & 43 & $\begin{array}{l}48.71 \pm 3.07 \\
\quad(\mathrm{EG})\end{array}$ & $\begin{array}{l}49.55 \pm 2.77 \\
\text { (CG) }\end{array}$ & $\begin{array}{c}10.95 \pm 1.59 \\
(\mathrm{EG}) ; \\
11.17 \pm 1.61 \\
(\mathrm{CG}) ; \\
\text { Difference between affected and not } \\
\text { affected side: } \geq 2 \text { to } \leq 8 \mathrm{~cm} ; \\
\text { Any other lymphedema conservative } \\
\text { treatment within a } 6 \text { months period; } \\
\text { CT/RT completed at } \leq 6 \text { weeks }\end{array}$ \\
\hline $\begin{array}{l}\text { El-Shazly et al } \\
(2016)(12)\end{array}$ & 60 & \multicolumn{2}{|c|}{$\begin{array}{c}\text { 30-50 years } \\
\text { Mean } \pm \text { SD not reported }\end{array}$} & Lymphedema stage II-III \\
\hline $\begin{array}{l}\text { Cebicci et al. } \\
(2016)(10)\end{array}$ & 11 & \multicolumn{2}{|c|}{$50.63 \pm 7.03$} & $\begin{array}{c}12(6-84) \text { months } \\
\text { Volume difference between affected and } \\
\text { not affected arm } \geq 200 \mathrm{ml} ; \\
\text { Circumference difference between } \\
\text { affected and not affected side: } \geq 2 \mathrm{~cm} ; \\
\text { Any other lymphedema conservative } \\
\text { treatment within a } 6 \text { months period; } \\
\text { CT/RT completed at } \leq 6 \text { months }\end{array}$ \\
\hline $\begin{array}{l}\text { Mahran et al. } \\
(2015)(12)\end{array}$ & 40 & $\begin{array}{c}52.13 \pm 4.0 \\
\text { (EG) }\end{array}$ & $\begin{array}{c}53.8 \pm 3.4 \\
\text { (CG) }\end{array}$ & $\begin{array}{c}15.6 \pm 2.82 \\
(\mathrm{EG}) ; \\
14.73 \pm 2.86 \\
(\mathrm{CG}) ; \\
\text { Circumference difference between } \\
\text { affected and not affected side: } \geq 2 \text { to } \leq 8 \\
\mathrm{~cm} ; \\
\text { Lymphedema elapsed } \geq 10 \text { months since } \\
\text { axilla node dissection }\end{array}$ \\
\hline $\begin{array}{l}\text { Bae et al (2013) } \\
\text { (14) }\end{array}$ & 7 & \multicolumn{2}{|c|}{$52 \pm 9.9$} & $\begin{array}{c}25.3 \pm 12.7 \\
\text { Lymphedema stage III; } \\
\text { Circumference difference between } \\
\text { affected and not affected side: } \geq 2 \mathrm{~cm} \text {; } \\
\text { Lymphatic obstruction confirmed by } \\
\text { lymphoscintigraphy }\end{array}$ \\
\hline
\end{tabular}

and also showing a certain heterogeneity in its method of evaluation: Mahran et al calculated both upper extremity circumferences at 10, 15 and $20 \mathrm{~cm}$ above and below the olecranon using a tape measurement, then calculating the summation of total differences between affected and healthy limbs at each measuring level (13); Bae et al measured upper extremity circumferences using a tape at $10 \mathrm{~cm}$ below the axilla and above the elbow, $7 \mathrm{~cm}$ below the elbow and above the wrist, then comparing with the measures of the not involved side 


\begin{tabular}{|c|c|c|c|c|c|c|}
\hline \multirow{7}{*}{ 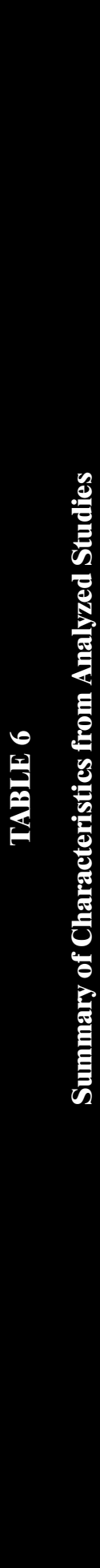 } & 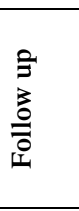 & 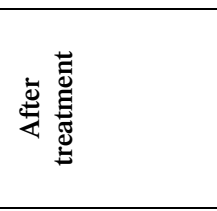 & 离莺 & 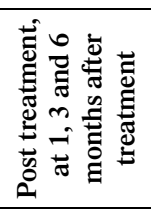 & 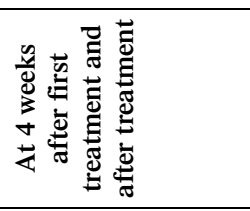 & 离莺 \\
\hline & 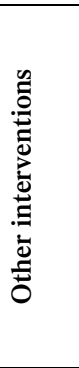 & 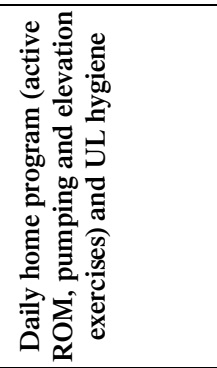 & 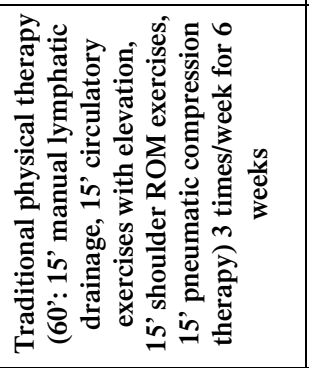 & 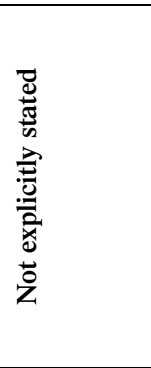 & 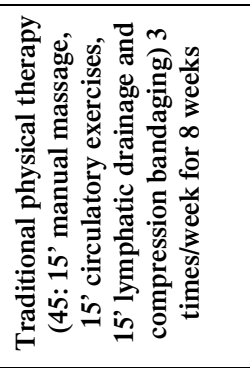 & 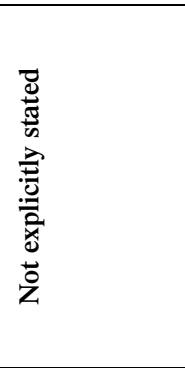 \\
\hline & 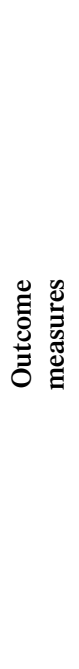 & 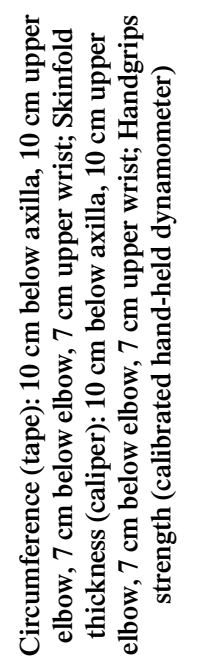 & 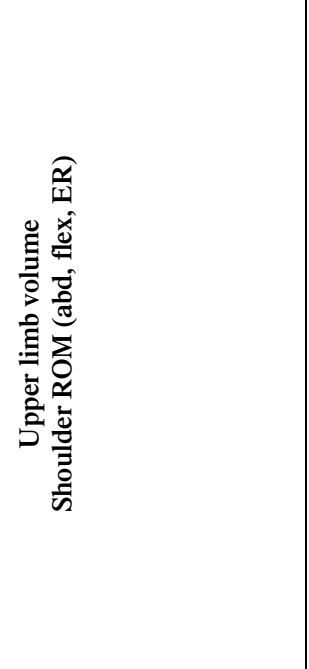 & 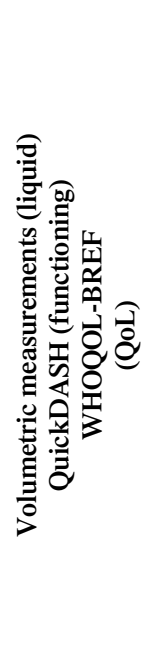 & 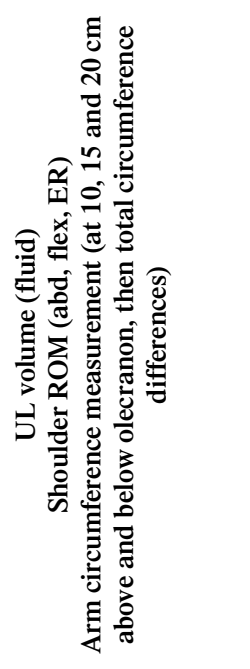 & 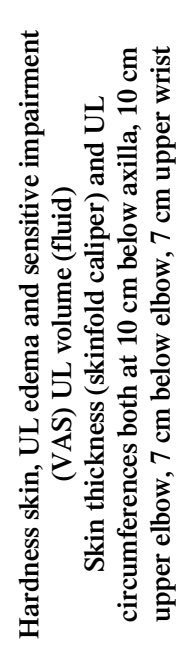 \\
\hline & 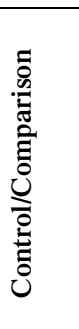 & 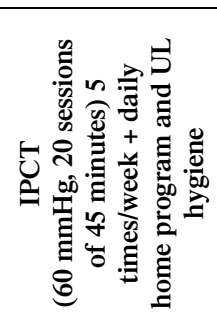 & 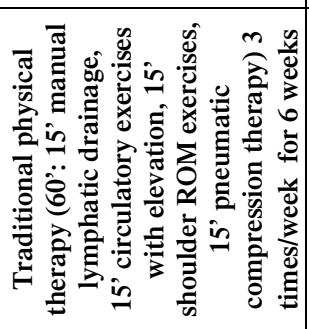 & 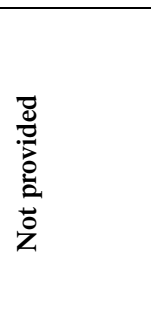 & 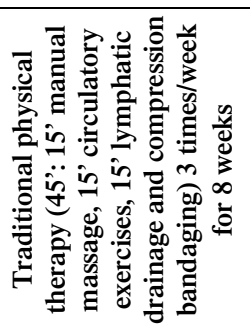 & 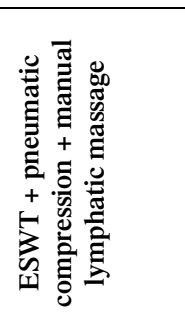 \\
\hline & $z$ & $\mathscr{q}$ & 8 & $=$ & q & $r$ \\
\hline & 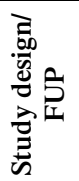 & 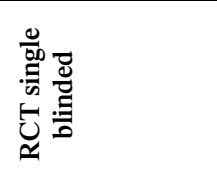 & $\underline{\widetilde{z}}$ & 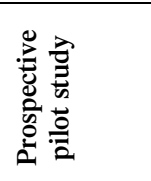 & $\underset{\approx}{t}$ & 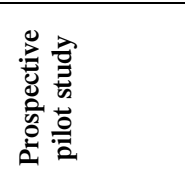 \\
\hline & 章 & 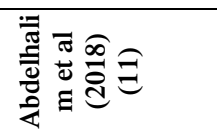 & 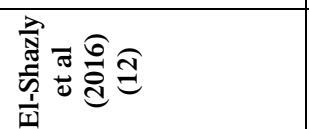 & 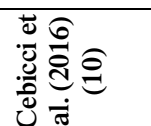 & 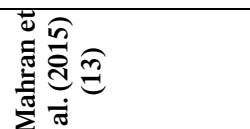 & 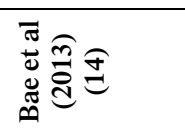 \\
\hline
\end{tabular}


(14); Abdelhalim et al evaluated affected upper extremity circumferences measuring them at $10 \mathrm{~cm}$ respectively below the axilla and above the elbow, at $7 \mathrm{~cm}$ respectively below the elbow and above the wrist, using a tape measurement and then calculating the mean values of three measurements performed at each level (11).

Regarding the ROM of the shoulder, this specific outcome was evaluated in only two studies $(12,13)$ and both authors evaluated flexion, abduction and external rotation of the shoulder. Mahran et al specified also that flexion and abduction were measured with the patient in standing position, while the latter was instead measured with patient in prone position, and all the measurements were performed by the same evaluator using a standard goniometer (13).

Two authors also evaluated minor objective measures of the disease: Bae et al measured skin thickness using a skinfold caliper at the aforementioned four anatomic levels, used for the upper extremity circumferences evaluation (14); and Abdelhalim et al evaluated instead handgrip strength, using a calibrated hand-held dynamometer according to Gomes et al protocol, and skin thickness, calculating the mean values of three measurements performed using a skinfold caliper at four anatomic levels of the upper extremity (the same used by authors for arm circumferences evaluation) (11).

Finally, two authors considered also subjective measures of lymphedema: Bae et al measured hardness of the skin, upper limb edema, and sensory impairment using the Visual Analog Scale (VAS), with 10 points being the most severe degree (14). Cebicci et al instead measured the functional status of the patients, using the Disability of the Arm, Shoulder and Hand Questionnaire - QuickDASH (10), and the quality of patient life, using the World Health Organization Quality of Life Assessment Questionnaire - WHOQOLBREF (10). (See Table 6 for details).

\section{Risk of bias in included studies}

Three of the five included trials were described as randomized. Abdelhalim et al specifies that an investigator not involved in the study conducted the randomization, utilizing sealed randomly filled envelopes including numbers of slips (11). El-Shazly et al and Mahran et al did not instead specify the method of randomization $(12,13)$. Allocation concealment was unclear in all the selected trials. Of the two prospective pilot studies, Bae et al reported that patients were divided into two groups by their opinion (14). Only one of the three RCTs reported information about blinding with Abdelhalim et al described their study design as a single blinded trial (11). Therefore Abdelhalim et al assumed also a study power of $95 \%$, a significant level of $5 \%$ $(\alpha=0.05)$ and an effect size of 1179 required at least a total sample of 34 subjects (11). A summary of the methodological quality assessment for each of the trials is described below:

Bae et al performed a prospective clinical trial in Korea including 7 patients affected by breast cancer-related upper limb stage III lymphedema. It was specified that subjects were assigned to the study group or control group by their opinion. It appears that allocation concealment was not adequate. No withdrawal or loss to follow-up was reported and although not explicitly stated (14). Mahran et al performed a randomized clinical trial in Saudi Arabia that included 40 post-menopausal women with post-mastectomy upper limb lymphedema. No information about randomization method, concealment of allocation, or study power was reported. It was not specified if outcome assessors were blinded to treatment. No withdrawal or loss to followup was reported (13). Cebicci et al performed a prospective clinical pilot study in Turkey and included 11 participants affected with a confirmed diagnosis of breast cancer and clinical manifestations of lymphedema. The study design did not provide a control group. No withdrawal or loss to follow-up was reported and although not explicitly stated (10). El-Shazly et al performed a randomized clinical trial in Egypt including 60 patients affected by unilateral lymphedema (stage 2,3 ) post-modified radical mastectomy or 
lumpectomy. The enrolled patients were described as randomized, however the method of randomization is unclear. It was not reported if concealment of allocation was adequate nor if outcome assessors were blinded to treatment. No information about study power was provided. No withdrawal or loss to follow-up was reported and although not explicitly stated (12). Abdelhalim et al performed a randomized controlled trial in Egypt including 48 participants affected by unilateral lymphedema post-breast cancer surgery. Patients in the trial were randomly assigned into two groups of equal number, each containing 24 subjects, utilizing sealed randomly filled envelopes from a bowl including number of slips with either number 1 or 2. However, it is unclear whether allocation concealment was adequate. It appears that patients and therapists were not blinded to treatment allocation while assessors blinding is reported. The study reported that a total number of 5 patients withdrew from the trial ( 3 in study groups and 2 in control group) and they were not included in the efficacy assessment (11). (Figs 2. and 3 summarize the risk of bias of the selected studies.)

\section{Effects of interventions}

For a more homogeneous analysis of the results, we decided to divide the studies according to their control group.

\section{Efficacy ESWT Versus Traditional Physical Therapy}

The two studies comparing ESWT treatment with traditional physical therapy $(12,13)$ observed a statistically significant reduction of mean arm volume difference as well as mean shoulder ROM for all the considered directions (flexion, abduction, external rotation) in the ESWT group when compared to the control group. Mahran et al also reported that mean of total circumferential difference were significantly reduced in the ESWT group when compared to the control group (13). One study instead compared results observed in patients treated with ESWT only and those emerged among patients treated with ESWT treatment in combination with lymphatic massage: it reported statistically significant improvement in the considered outcomes in both groups, however, a comparison of the two groups did not show a significant difference (14).

\section{ESWT Versus Intermittent Pneumatic Compression Therapy}

Abdelhaim et al reported significant differences in favor of ESWT in the considered endpoints (upper limb circumferences and skin thickness), except for handgrips strength that showed instead non-significant post-treatment differences between both sides and both groups (11).

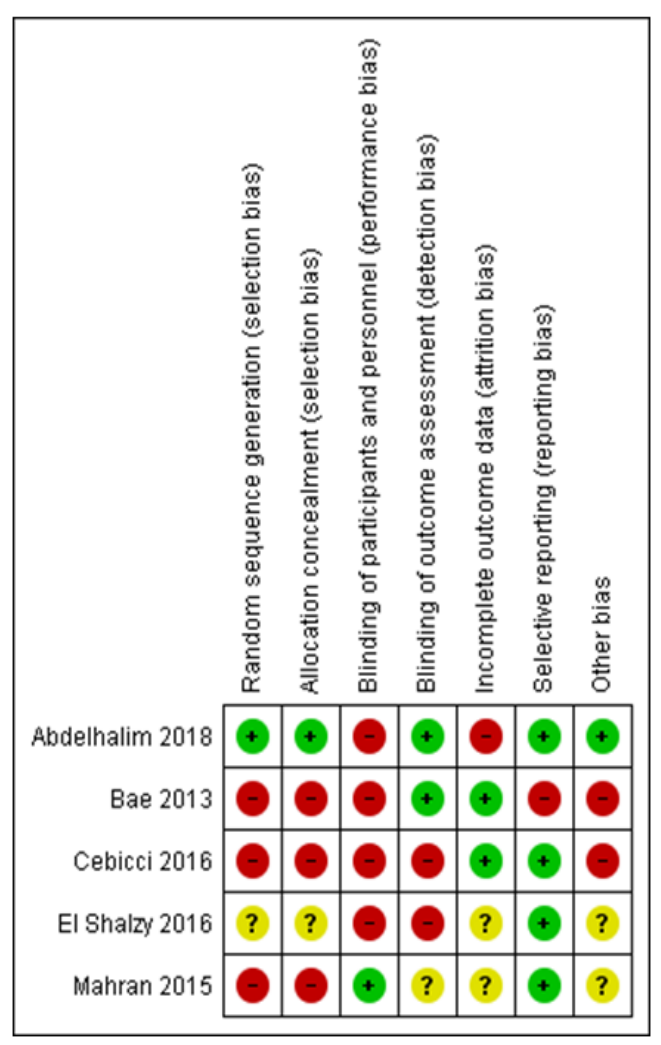

Fig. 2. Identified risk of bias for single studies 


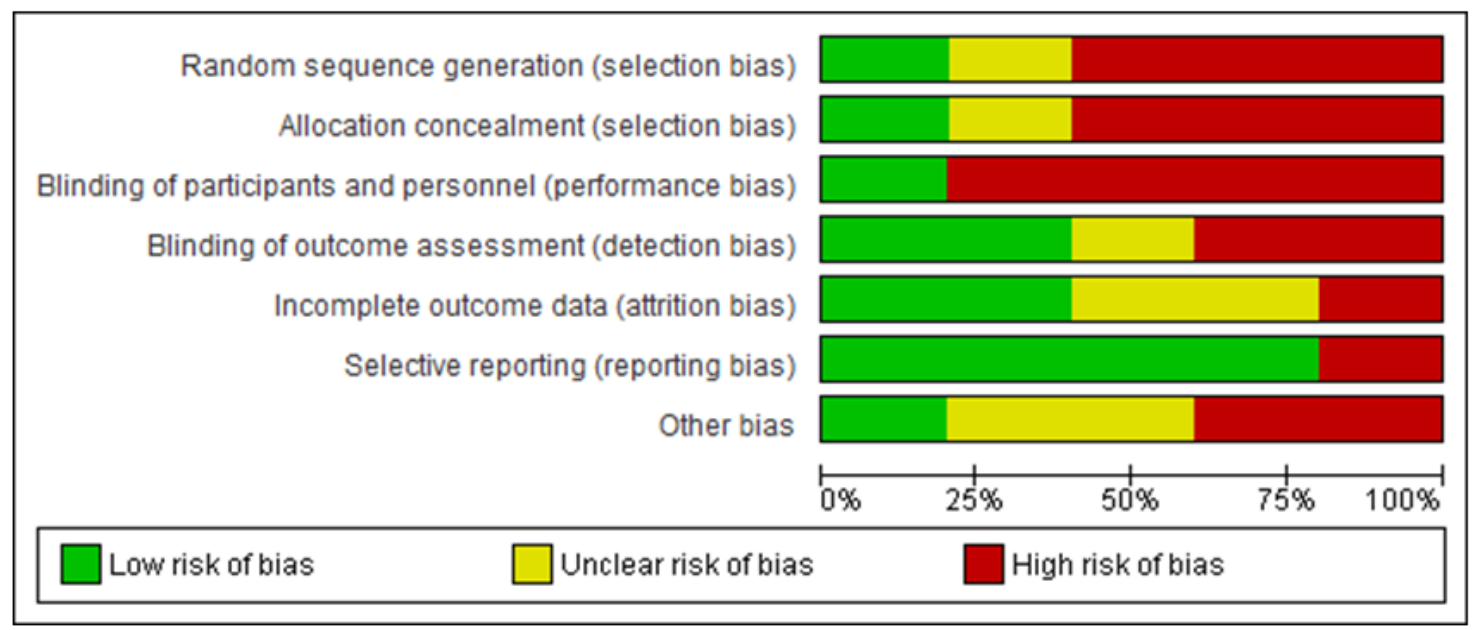

Fig. 3. Global identified risk of bias for selected studies

\section{ESWT Treatment Only}

Cebicci et al reported significantly reduction in the amount of lymphedema with ESWT treatment and it was maintained for 6 months. Results observed in improving volumetric measurements and functional outcomes were statistically significant when compared to those reported in the previous timing of follow-up assessments. A statistically significant improvement was seen also in the scores for the "physical health domain" comprised in the WHOQOL-BREF reported at each time of follow-up when compared to baseline. However, there was no significantly improvement in this specific outcome when comparing post-treatment and mid-term follow-up assessments (10).

\section{Adverse Effects}

Cebicci et al reported transient redness of the skin in two patients at the ESWT application site (10). No other adverse effects were reported in other studies.

\section{DISCUSSION}

Prior to discussing the general conclusions, it is useful to briefly recapitulate the main observations from the study of the selected trials from the analysis of the scientific literature about the application of ESWT in breast cancer-related lymphedema. For a more homogeneous discussion, we divided them based on the characteristics of the control group.

\section{ESWT Versus Traditional Physical Therapy}

El-Shazly et al (12) designed a randomized controlled trial of medium-low quality that included 60 participants and compared ESWT treatment with traditional physical therapy. Regarding the evaluated outcomes (arm volume, shoulder ROM), it reported significant differences in favor of ESWT group. However, the encouraging results observed in the experimental group must be contextualized with the limits that emerged from the analysis of the study, such as the presence of co-intervention and the poor follow-up. Furthermore, a lack of accuracy in the description of the materials and methods was found. While authors properly described the studied population characteristics (including the precise stage of lymphedema and the exclusion criteria) and the ESWT setting parameters, they provided no accurate information about the evaluation of clinical outcome measurements nor the site of application of ESWT therapy on the 
affected upper limb. Mahran et al (13) designed a randomized clinical trial of medium-low quality, including 40 participants and compared ESWT treatment with traditional physical therapy. As with the previous study, a certain heterogeneity in the accuracy of the description of the main constituent elements of the trial was found. In fact, although authors properly described both the studied population (including timing of symptoms and exclusion criteria) and outcome measurements methods (upper extremity circumferences, arm volume and shoulder ROM), only a partial description of the ESWT setting parameters was given, as the site of application of the ESWT therapy on the treated upper extremities was not reported. These observations, combined with the evidence of a poor follow-up and the presence of co-intervention, must inevitably take into consideration the statistically significant differences observed in the results of the experimental group compared to the control group.

Finally, Bae et al (14) designed a prospective clinical trial, including 7 participants and comparing ESWT treatment only with ESWT treatment combined with lymphatic massage. Although only a partial description of the method of application of ESWT therapy was reported, the authors properly described the main constituent elements of the trial (study population characteristics, ESWT setting parameters, and method of clinical outcome measurements evaluation). They reported a statistically significant improvement in the considered outcomes (upper extremity volume, upper extremity circumferences and skin thickness) in both groups, however, a comparison of the two groups did not show a significant difference. Finally, these results must be contextualized with the limitations of the study: poor sample population, study design (no randomized controlled trial model), and an outcome assessment performed only after completion of the treatment.

Regarding the strengths and limits of the aforementioned studies, it is necessary to consider their comparison. First, despite the similar characteristics of the study population, the ESWT treatment protocol and the type of control group, a substantial lack of uniformity in the evaluated outcomes was found. This considerable variability allowed only a descriptive analysis of the data, strongly limiting any comparison. Secondly, regarding interpretation of the results, although a statistically significant clinical improvement was observed in the experimental groups, these encouraging results must be contextualized within the limitations of the aforementioned studies. The medium-high risk of bias due to the methodology used, the poor follow up, and the co-intervention (if present) inevitably reduced the reliability of the observed results.

\section{ESWT Versus Intermittent Pneumatic Compression Therapy}

The study conducted by Abdelhaim et al is a high-medium quality single-blinded randomized clinical trial, including 48 participants and comparing ESWT to intermittent pneumatic compression therapy (11). As for the aforementioned studies, the description of the study population, both for clinical lymphedema characteristics and time interval from previous treatments, was exhaustive. ESWT treatment parameters were properly reported as well, including the specified site of pulse application. Regarding clinical outcomes (upper extremity circumferences, skin fold thickness, handgrip strength), measurements were performed on both sides and accurately described. Side effects were recorded. Recommendations for a daily home program and hygiene and skin care were provided. The post-treatment evaluation reported improvement in all outcomes, however, significant differences in favor of ESWT were observed in all endpoints, except for the handgrip strength that showed instead non-significant post-treatment differences between both sides and for both groups. Although the conduct of the whole trial was fair, the poor follow up is a significant limitation.

\section{ESWT Treatment Only}


Finally, Cebicci et al performed a prospective clinical pilot study, including 11 participants that evaluated ESWT treatment only efficacy without providing a control group (10). As for the previous studies, the description of the study population was exhaustive. ESWT treatment parameters were properly reported, including the specific site of pulse application. No co-intervention appears to have been performed. The authors divided outcome measures into primary (arm volume) and secondary (functional status, quality of life). Outcome assessment was performed immediately after completing the treatment and at 1, 3 and 6 months after last treatment. A statistically significant improvement in outcomes was found compared to those reported in the previous timing of follow-up assessments except for the "physical health" domain in the WHOQOL-BREF when comparing post-treatment and mid-term follow-up assessment. Despite the fair quality of conduct of the study including the exhaustive information about the study population and ESWT parameters and method of application, the non-comparative study design precluded any comparisons with other treatments methods. The lack of a control group, combined with a small sample size and only one objective measure of lymphedema progression, inevitably limited the study conclusion. However, the long-term follow-up and evaluation of the quality of life (including the functional/disability status of the patients) are two strengths, especially when comparing with the previously described trials.

According to these observations, a lack of uniformity about different elements of the selected studies is evident. First, regarding the studied population, even if participants were described in their characteristics in all the aforementioned trials, inclusion criteria chosen by authors often differed from each other, and in some cases were not precisely reported. For a more reliable interpretation of the results, an accurate description of participants should be always reported (in particular: age, stage of lymphedema, previous surgery, and adjuvant therapies). Moreover, in order to increase the reliability of the study, authors should report also the "cut off" range used to select population in terms of "lymphedema stage" (e.g., upper extremities circumferences difference) and "timing from the last adjuvant treatment". For the same purpose, any cointervention administered during the whole treatment and/or follow-up period should be properly reported and described in detail as well.

Secondly, a certain variability in the used ESWT treatment modality among the selected studies was also found. Despite the authors choosing quite similar ESWT setting parameters, a substantial heterogeneity in the method and the timing of application has emerged through our analysis. If the lack of some information about the ESWT treatment modality is attributable to the approximation with which some authors describe the treatment provided, the heterogeneity of the used approach is likely due to the lack of a standard shared protocol for the use of ESWT in the treatment of lymphedema. Plus, in view of the contribution of the ESWT to the breast cancerrelated lymphedema traditional therapy, primarily supported by "in vitro/ animal model" studies, a shared standard protocol about setting ESWT parameters and method in the treatment of this specific affection would certainly be useful for the practical clinic.

Concerning the outcomes measurement, the profound variability observed among the selected studies was the major element which affect the reliability in the comparison of the results. This discrepancy was detected not only in the outcome measures chosen by authors, but also about the specific measurement method of the same outcome. In our opinion, to facilitate judgment about clinically important differences between treatment groups, first it would be useful to have a consensus about what constitutes a clinically important improvement in a patient with breast cancer-related lymphedema. In support of this, an international consensus for the use of a standard set of objective and subjective outcome measures in clinical trials for breast cancer-related lymphedema, which are valid and reliable, would improve our ability to 
interpret and compare results. These might include arm volume, a measurement of upper extremity circumferences (specifying the site of measurements), affected shoulder range of motion (specifying if passive or active), and an easily repeatable functioning scale, possibly including a measure of quality-of-life. Finally, regarding the timing of follow up, except for one study (9), an inadequate assessment took place. Considering the often chronic and recrudescent characteristics of lymphedema, an only after treatment outcome assessment is clearly insufficient. Moreover, according to the biological times necessary to establish the effectiveness of shock wave therapy, a followup should be performed at least 8 weeks after the last treatment.

The discrepancies and variability of the previously described elements, make it difficult not only to compare results among the different studies but also to assert with confidence the effects, and further the possible superiority of ESWT in the management of breast cancer-related lymphedema when compared to other conservative therapies. Despite the improvement of the considered outcomes emerging in our review, it is clear that the effects of ESWT in the treatment of breast cancer-related lymphedema should be further evaluated in high quality RCTS, taking into account the weaknesses and strengths of the studies conducted to date.

\section{Implication for practice:}

According to what emerged from the analyzed trials and the biological effects detected among "in vitro/animal model" studies, this review encourages the further use of ESWT for breast cancer-related lymphedema in support of the gold standard treatment (CDPT, home-based exercises program, skin care, and hygiene protocol). In fact, according to the progressive and often dramatic evolution of the pathology, the use of a safe, effective, economical and easily repeatable treatment method, with proven biological effects as the ESWT, could significantly enhance the benefits of the already widely validated traditional therapy. This approach might reduce the impact of the disease on the quality-of-life of the affected patients and its complications (e.g., lymphangitis, deep venous thrombosis). However, our review recognizes that further research is needed to properly investigate first, the midlong term effects of ESWT treatment, and secondly, its possible superiority over other conservative therapies with side effects or contraindications.

\section{Implication for research:}

New high-quality RCTs evaluating the effects of ESWT in patients affected by breast cancer-related lymphedema are needed. Trials should have a low risk of bias, including in its report: the method of randomization, treatment allocation concealment and blinding, the study power, and an intention to treat analysis. Trials should properly describe the selected population, including type of surgery, stage of lymphedema, duration of symptoms, and timing from other eventual treatments. A complete description of ESWT treatment parameters, site and method of application should be reported. Any other treatment provided prior or during interventions/followup assessments should be reported and properly described. Due to the natural history of breast cancer-related lymphedema, trials should include at least medium-term follow-up. Development of a standard set of clinically important, valid and reliable outcome measures for the improvement of the pathological conditions, would significantly improve the comparison between among studies.

\section{ACKNOWLEDGMENTS}

The authors received no financial support for the research, authorship, and/or publication of this article.

\section{CONFLICT OF INTEREST AND DISCLOSURE}

The authors declare no competing financial interests exist. 


\section{REFERENCES}

1. Bland, KI, EM Copeland, VS Klimberg et al: The Breast. Comprehensive Management of Benign and Malignant Diseases, 5th Edition. Elsevier, US, 2018.

2. The Diagnosis and Treatment of Peripheral Lymphedema: 2020 Consensus Document of the International Society of Lymphology. Lymphology 53 (2020) 3-19.

3. Földi, M, E Földi, RHK Strobenreuther, S Kubik (Eds): Textbook of Lymphology, 3rd Edition. Elsevier, Munich, 2012.

4. Armer, JM, JL Feldman, PL Ostby, et al: Simplifying evidence-based management of breast cancer-related lymphedema. Expert Review of Quality of Life in Cancer Care 1(5) (2016), 389-402.

5. Szolnoky, G, A Dobozy, L Kemény, et al: (Eds): Towards an effective management of chronic lymphedema. Clinics in Dermatology. 32 (2014), 685-691.

6. Kim, IG, JY Lee, DS Lee et al: Extracorporeal shock wave therapy combined with vascular endothelial growth factor-C hydrogel for lymphangiogenesis. J. Vasc. Res. 50 (2013), 124-133.

7. Kubo, M, TS Li, T Kamota, et al: Extracorporeal shock wave therapy ameliorates secondary lymphedema by promoting lymphangiogenesis. J. Vasc. Surg. 52 (2010), 429-434.

8. Serizawa, F, K Ito, M Matsubara, et al: Extracorporeal shock wave therapy induces therapeutic lymphangiogenesis in a rat model of secondary lymphoedema. Eur. J. Vasc. Endovasc. Surg. 42 (2011), 254-260.
9. Kuo, YR, CT Wang, FS Wang, et al: Extracorporeal shock-wave therapy enhanced wound healing via increasing topical blood perfusion and tissue regeneration in a rat model of STZ-induced diabetes. Wound Repair Regen. 17 (2009), 522-530.

10. Cebicci, MA, ST Sutbeyaz, SS Goksu, et al: Extracorporeal shock wave therapy for breast cancer-related lymphedema: A pilot study. Arch. Phys. Med. Rehabil. 97 (2016), 15201525.

11. Abdelhalim, NM, AF Samhan: Comparison of extracorporeal shock waves therapy versus intermittent pneumatic compression therapy in breast cancer-related lymphedema. Int. J. Cancer Res. 14 (2018), 77-85.

12. El-Shazly, M, WH Bahrain, WN Thabet et al: Response of post-mastectomy lymphedema to extracorporeal shockwave therapy. J. Surg. 4 (2016), 14-20.

13. Mahran, HG, AA Thabet: Extracorporeal shockwave therapy for post-menopausal patients with breast cancer-related lymphedema. Int. J. Cancer Res. 49 (2015), 2051-2784.

14. Bae, H, HJ Kim: Clinical outcomes of extracorporeal shock wave therapy in patients with secondary lymphedema: A pilot study. Ann. Rehabil. Med. 37 (2013), 229-234.

\author{
Dr. Federica Bressi \\ Physical and Rehabilitative Medicine \\ Università Campus Bio-Medico di Roma \\ Via Alvaro del Portillo 5 \\ 00128 Roma (Italia) \\ Phone: 0039-6-22541615; 0039-3398253221 \\ Fax: 00396-22541602 \\ E-mail: f.bressi@unicampus.it
}

